This item was submitted to Loughborough's Research Repository by the author.

Items in Figshare are protected by copyright, with all rights reserved, unless otherwise indicated.

\title{
The i-DREAMS intervention strategies to reduce driver fatigue and sleepiness for different transport modes
}

PLEASE CITE THE PUBLISHED VERSION

https://doi.org/10.1109/MT-ITS49943.2021.9529340

PUBLISHER

IEEE

VERSION

AM (Accepted Manuscript)

\section{PUBLISHER STATEMENT}

Personal use of this material is permitted. Permission from IEEE must be obtained for all other uses, in any current or future media, including reprinting/republishing this material for advertising or promotional purposes, creating new collective works, for resale or redistribution to servers or lists, or reuse of any copyrighted component of this work in other works.

\section{LICENCE}

\section{All Rights Reserved}

\section{REPOSITORY RECORD}

Pilkington-Cheney, Fran, Amir Pooyan Afghari, Ashleigh Filtness, Eleonora Papadimitriou, André Lourenço, and Tom Brijs. 2021. "The I-dreams Intervention Strategies to Reduce Driver Fatigue and Sleepiness for Different Transport Modes”. Loughborough University. https://hdl.handle.net/2134/14974119.v1. 


\section{The i-DREAMS intervention strategies to reduce driver fatigue and sleepiness for different transport modes}

\author{
Fran Pilkington-Cheney \\ Transport Safety Research Centre \\ Loughborough University \\ Loughborough, UK \\ 0000-0001-8043-3137 \\ Eleonora Papadimitriou \\ Safety and Security Science Section \\ Delft University of Technology \\ Delft, The Netherlands \\ E.papadimitriou@tudelft.nl
}

\author{
Amir Pooyan Afghari \\ Safety and Security Science Section \\ Delft University of Technology \\ Delft, The Netherlands \\ a.p.afghari-1@ tudelft.nl \\ André Lourenço \\ CardioID Technologies \\ Instituto Superior de Engenharia de \\ Lisboa, Portugal \\ arl@cardio-id.com
}

\author{
Ashleigh Filtness \\ Transport Safety Research Centre \\ Loughborough University \\ Loughborough, UK \\ 0000-0001-7357-0344 \\ Tom Brijs \\ Transportation Research Institute \\ Hasselt University \\ Diepenbeek, Belgium \\ 0000-003-2622-4398
}

\begin{abstract}
Driver sleepiness and fatigue are important contributors to many transport incidents and significantly increase crash risk. Recently, detection systems have been developed which aim to monitor the state of the driver and detect increasing levels of fatigue. However, there has been less focus on appropriate intervention strategies for drivers once fatigue and sleepiness have been detected. This paper describes the i-DREAMS fatigue intervention strategies, which aim to keep drivers within a safe driving zone. Interventions will be provided both in real-time and post-trip and can be customized to be used with a variety of transport modes. Real-time interventions will measure fatigue through trip duration, and driver sleepiness through heart-rate variability (HRV) information, obtained by means of sensors in the steering wheel or a wearable device, and attributed to Karolinska Sleepiness Score (KSS) bands. Thresholds for warnings will map onto phases of a 'Safety Tolerance Zone' and will be dynamic changing as the driver state and driving situation develops. Posttrip interventions will aggregate data throughout the duration of the drive and aim to provide customized feedback and coping tips related to driver levels of fatigue and sleepiness, to improve driving behavior. Goals and challenges will add a gamified aspect to the post-trip interventions. The next stage of the development of the i-DREAMS fatigue intervention strategy is to test the concept in a series of simulator and field trials. Future research should explore acceptance and compliance of interventions and frequency of alerts.
\end{abstract}

Keywords-driver sleepiness, fatigue, transport safety, driver monitoring, interventions

\section{INTRODUCTION}

Driver sleepiness and fatigue are significant risk factors for motor vehicle incidents [1]. Developing effective fatigue and sleepiness detection systems and appropriate intervention strategies may improve safety by warning drivers of potential performance impairment. There has been recent interest in developing systems which detect sleepiness and fatigue, however, there has been less discussion relating to appropriate intervention strategies and timing of interventions once fatigue or sleepiness has been detected. This paper aims to explore potential real-time and post-trip intervention strategies for fatigue and sleepiness as part of a context aware

Research funded by the EU-HORIZON 2020 research and innovation program (Grant Agreement No.: 814761) driver assistance platform which aims to keep drivers within the boundaries of safe driving.

\section{A. Definition of fatigue and sleepiness}

Within occupational settings, the terms fatigue and sleepiness are often used interchangeably. In transportation, fatigue is generally the preferred term, however, within the literature, fatigue and sleepiness have distinct definitions. Where fatigue is typically an inability to continue with a task which has been continuing too long [2], and can be influenced by task duration, monotony and workload (both under- and over-load), sleepiness is defined as a physiological urge to fall asleep caused by lack of or disturbed sleep and the body's circadian rhythms [3]. As fatigue and sleepiness have different mechanisms, they require different indicators and methods of detection.

\section{B. Effects of fatigue and sleepiness on crash risk}

When sleepy or fatigued, drivers show decrements in performance associated with simple and complex tasks, impaired attention, slowed reaction times and loss of conscious awareness behind the wheel [4], all crucial aspects for driving. Sleepiness and fatigue also result in a higher rate of lane crossings [5], reduced hazard perception [6] and an increased tendency to become distracted [7]. Therefore, driving when sleepy or fatigued can have severe consequences, and increases potential crash risk [1]. Sleepiness risk can often be underestimated. Most people are aware when they are sleepy but may not have accurate insight or belief about how this will affect their driving performance. Therefore, they may continue driving when they are at unsafe fatigue or sleepiness levels. Systems which can monitor and detect sleepiness and fatigue, and provide appropriate interventions therefore have the potential to reduce the risk of individuals being involved in a sleepiness or fatigue related crash.

\section{Detecting fatigue and sleepiness}

Fatigue and sleepiness can be measured in a variety of different ways, both subjectively and objectively. However, it is important to consider indicators which can be applied in the context of driving. In relation to fatigue, time spent on task has been shown to impact and increase fatigue levels [8]. Therefore, duration of driving can be used as an indicator of 
fatigue. In terms of sleepiness detection, ocular measures can be used in commercial detection technology systems. Many ocular measures are associated with sleepiness, such as blink frequency and duration, eyelid closure, and pupil dimension [9]. However, there can be issues with ocular detection, for example head position and movements, as well as poor lighting can affect accuracy. They also typically involve the recording of individuals which can be met with resistance in a professional setting.

With technological advancements in sensors, physiological data is becoming more easily accessible, which provides other options for non-intrusive sleepiness detection. Recently heart rate and heart rate variability (HRV) based measures have been investigated as methods of sleepiness detection $[10,11]$. HRV has not been overly utilised in commercial detection systems and it has been suggested that HRV requires further research and validation, in particular to control for confounding factors that influence heart rate [10]. However, heart rate signals show potential to detect increasing levels of sleepiness and therefore to be incorporated into detection systems, particularly as sensors can be embedded within wearables [12], seats or steering wheels [13].

\section{Interventions for fatigue and sleepiness}

In relation to interventions, the first problem is knowing when the best time is to intervene, (e.g., at what level of driver sleepiness), and doing so in a flexible manner that is suitable for the situation and transport mode. The second problem is providing a suitable and effective intervention. Different transport modes have different opportunities for intervention, for instance bus and rail drivers are operating to a fixed passenger schedule. It would therefore not be appropriate for a bus driver to stop and take a break or nap in the same way a private car driver could. On the other hand, professional drivers are restricted in their driving hours by regulations which do not apply to private car drivers. In terms of the timing of the intervention, fatigue or sleepiness warnings need to be preventative, in that they should be given in enough time for the driver to take action. Interventions may also need to change as the driving situation develops. Therefore, developing real-time and post-trip interventions for fatigue and sleepiness need to take into consideration not only the driver state and driving context, but also the transport mode and the appropriate level of intervention.

\section{OBJECTIVES AND APPROACH}

\section{A. Objectives}

The recent technological developments in collecting detailed data about drivers' fatigue and sleepiness have created new opportunities for the design of customized interventions to mitigate the risk caused by these two factors. This study aims to design a framework for leveraging the collected data and develop strategies for applying real-time and post-trip interventions for different transport modes, in order to reduce driver's fatigue and sleepiness and keep them in safe driving conditions.

\section{B. Study context-i-DREAMS}

This work is part of the EU-funded Horizon 2020 iDREAMS project, which aims to develop, test and validate a context aware safe driving platform, taking into account driver related background factors, risk related real-time physiological indicators and driving task complexity, to determine if a driver is within the boundaries of safe operation. Interventions (both real-time and post-trip) will be applied if the driver reaches the limits of the safe driving zone, for example if the platform determines the driver to be fatigued or sleepy. i-DREAMS aims to take a holistic approach in driver monitoring, and one aspect considered is fatigue and sleepiness, which forms the basis of the current paper. For the purpose of the project, the i-DREAMS fatigue intervention strategy incorporates both fatigue and sleepiness. The strategy is based on the duration of the drive (an indicator of fatigue) and then adjusted in relation to driver sleepiness. Sleepiness is detected through sensors embedded in either a steering wheel or a wearable, which measures heart rate in a 2 minute rolling window. An algorithm is then applied which converts the data into Karolinska Sleepiness Score (KSS) bands, which indicates the sleepiness state of the driver. The KSS [14] is a subjective measure of sleepiness, consisting of a 9-point scale ranging from 1, extremely alert to 9 , very sleepy, great effort to keep awake, fighting sleep. A KSS score of 7 or above is associated with sleepiness, with level 8 and 9 being critical. The project intends to detect and provide interventions for 4 transport modes: car, bus, truck and rail.

\section{Introducing the Safety Tolerance Zone}

Within the i-DREAMS project, the safe driving zone has been conceptualized as the 'Safety Tolerance Zone' (STZ), which has three phases. A normal driving zone phase where the crash risk is minimal, for example the driver is alert; a danger phase where the risk of a crash increases as events occur external to or within the vehicle (driver sleepiness or time on task increases); and an avoidable crash phase (for example when the driver is experiencing a high level of sleepiness). This last phase is where a crash is very likely to occur if no preventative action is taken by the driver or another vehicle driver. The system will monitor the driver and various driving context elements, and if the drivers enters the danger phase, a warning will be triggered, which will develop if the driver takes no corrective action. The phases of the STZ are deemed to be dynamic and constantly changing and updating. Therefore, if the driver enters the danger phase, and acts on the warning, for example by taking a break, having caffeine or a nap, then this may transition the driver back in the normal driving phase.

\section{I-DREAMS INTERVENTION STRATEGY}

A pivotal element of the i-DREAMS project following the definition and development of the STZ is the application of safety-oriented interventions in order to reduce risk (fatigue and sleepiness in this context) among drivers of different transport modes and keep them in a safe driving state. The real-time interventions are implemented in the vehicle and aim to keep the driver in a safe (immediate) driving stage (e.g. the first level of the STZ) whereas the post-trip interventions are implemented after the trip has been completed and aim to provide feedback to drivers about their driving behaviour, help them identify their risky behaviours and encourage them to develop safe driving behaviours and attitudes.

Effectiveness and acceptance are two crucial properties of the interventions that need to be considered prior to their 
design and implementation. Fatigue interventions, for example, should be effective enough to prevent fatigue during the drive and at the same time should be acceptable enough to be well received by the drivers. Following a comprehensive review of several acceptance theories in the literature including the technology acceptance model, theory of planned behaviour, unified theory of acceptance and use of technology, unified model of driver acceptance, and transtheoretical model of change (please refer to [15] for a discussion on these theories), the real-time and post-trip fatigue interventions are proposed as follows.

\section{A. Real-time fatigue intervention}

Real-time fatigue interventions (in-vehicle) are triggered when fatigue is detected during the drive. These interventions can take the form of a visual warning and/or an auditory alarm and can be implemented through an external in-vehicle intervention device that is already installed in the vehicle for other purposes. It is worth re-iterating that sleepiness and fatigue are both considered in the i-DREAMS project as separate but closely interlinked risk factors to be addressed using real-time interventions. As a result, the fatigue intervention strategy needs to be adjusted with respect to sleepiness as well.

The real-time fatigue interventions may include a visual warning, possibly a symbol of a coffee cup that would indicate planning for a break and taking a short nap. A schematic of such a visual warning which aims to make drivers aware that they need to monitor their performance is shown in Fig. 1. This visual warning may be accompanied by an auditory alert as the severity of the risk indicators (KSS score and drive duration for sleepiness and fatigue, respectively) increases, giving the indication to the drivers that they need to take immediate action and stop driving at the earliest opportunity.

The notion of intervening differently at the distinct stages of the STZ requires defining thresholds to distinguish the three stages in each risk factor (i.e., the KSS score and the duration of the drive). In doing so, it is also important to consider early versus late timing of interventions. An early intervention provides sufficient time for the driver to take evasive actions but may result in false-positive warnings and distract the driver [16]. A late intervention, on the other hand, is less prone to false-positive warnings but may be too late for taking evasive actions. It has been shown that it is more beneficial for drivers to be informed early, yet in a non-intrusive way [17]. Taking these considerations into account, a general definition of these thresholds which are primarily defined to correlate fatigue with the three stages of the safety tolerance zone are shown in TABLE I.

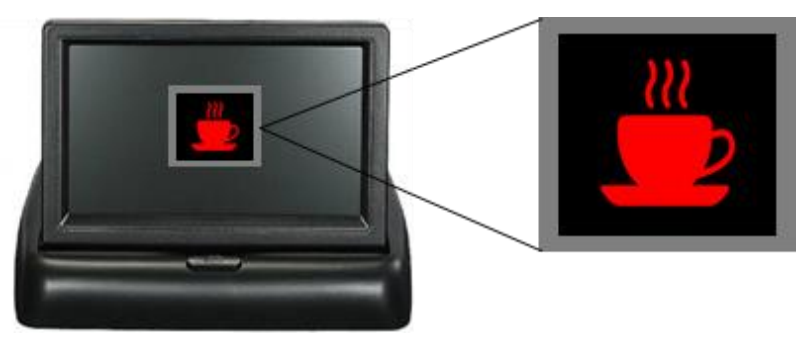

Fig. 1. Proposed fatigue warning and its visual representation
TABLE I. Proposed real-time intervention strategy for fatigue

\begin{tabular}{|c|c|c|c|}
\hline $\begin{array}{c}\text { Drive } \\
\text { duration } \\
(\mathrm{t}) \\
\end{array}$ & Warning suggestion & Meaning & STZ \\
\hline $\begin{array}{l}\mathrm{t} \text { below } \\
\mathrm{h}_{1} \text { hours }\end{array}$ & \multicolumn{2}{|c|}{ No warning } & $\begin{array}{l}\text { Normal } \\
\text { driving }\end{array}$ \\
\hline $\begin{array}{c}\mathrm{t} \text { above } \\
\mathrm{h}_{1} \text { hours } \\
\text { but } \\
\text { below } \mathrm{h}_{2} \\
\text { hours }\end{array}$ & $\begin{array}{l}\text { Visual warning, cup of } \\
\text { coffee symbol indicating } \\
\text { plan for a break. }\end{array}$ & $\begin{array}{l}\text { Make drivers } \\
\text { aware they } \\
\text { need to think } \\
\text { about planning } \\
\text { a break due to } \\
\text { 'fatigue'. }\end{array}$ & $\begin{array}{c}\text { Danger } \\
\text { zone }\end{array}$ \\
\hline $\begin{array}{c}\mathrm{t} \text { above } \\
\mathrm{h}_{2} \text { hours } \\
\text { but } \\
\text { below } \mathrm{h}_{3} \\
\text { hours }\end{array}$ & $\begin{array}{l}\text { Visual warning, may } \\
\text { change colour } \\
\text { accompanied by an } \\
\text { auditory alert. }\end{array}$ & $\begin{array}{l}\text { Drivers need to } \\
\text { stop at the } \\
\text { nearest } \\
\text { opportunity. }\end{array}$ & \multirow{2}{*}{$\begin{array}{l}\text { Avoidable } \\
\text { crash zone }\end{array}$} \\
\hline $\begin{array}{c}\mathrm{t} \text { above } \\
\mathrm{h}_{3} \text { hours }\end{array}$ & $\begin{array}{c}\text { Increased } \\
\text { infrequency/pitch/volume } \\
\text { of warning. Visual } \\
\text { symbol may flash. }\end{array}$ & $\begin{array}{l}\text { Drivers must } \\
\text { stop at the } \\
\text { nearest } \\
\text { opportunity. }\end{array}$ & \\
\hline
\end{tabular}

For project confidentiality reasons, the exact thresholds for different parameters are not shown in this manuscript. It is noted that initial values for these thresholds are used and the thresholds will be refined based on preliminary data analysis from i-DREAMS pilot tests. These thresholds, however, should be adjusted based on the sleepiness state of the driver, indicated by KSS score. As drivers become sleepier, the drive duration thresholds for fatigue warnings should decrease. The general sleepiness thresholds for this purpose are presented in TABLE II. According to these thresholds, the KSS score lower than $\mathrm{K}_{\mathrm{a}}$ is associated with the "normal" phase of STZ, the KSS score between $\mathrm{K}_{\mathrm{a}}$ and $\mathrm{K}_{\mathrm{b}}$ is associated with the "danger" phase of the STZ and the KSS score of more than $\mathrm{K}_{\mathrm{b}}$ is associated with the "avoidable crash" phase of the STZ.

A real-time fatigue intervention strategy is now designed based on the thresholds of the drive duration, adjusted by the thresholds of the KSS score. In doing so, it is assumed that the adjustment increments are in line with the increments of the KSS score.

Fig. 2 presents the proposed fatigue intervention strategy for passenger cars. According to section (b) in Fig.2, no warning is given to the drivers if the drive duration is less than $\mathrm{h}_{1}$ hours and the KSS score is less than $\mathrm{K}_{\mathrm{a}}$. This state is considered to be the "normal" phase of the STZ. A visual warning is given to the drivers if the drive duration remains less than $h_{1}$ hours but the KSS score is between $K_{a}$ and $K_{b}$. This state is considered to be the "danger" phase of the STZ.

TABLE II. Proposed thresholds linking the KSS score with the STZ

\begin{tabular}{|c|c|}
\hline KSS scale & STZ \\
\hline $\begin{array}{c}\text { KSS }<\mathrm{K}_{\mathrm{a}} \\
\text { (driver is either sufficiently alert or } \\
\text { neither alert nor sleepy) }\end{array}$ & Normal driving \\
\hline $\begin{array}{c}\mathrm{K}_{\mathrm{a}}<\mathrm{KSS}<\mathrm{K}_{\mathrm{b}} \\
\text { (driver shows some signs of sleepiness, } \\
\text { but no effort to keep awake) }\end{array}$ & Danger zone \\
\hline $\begin{array}{c}\text { KSS }>\mathrm{K}_{\mathrm{b}} \\
\text { (driver is very sleepy, fighting } \\
\text { sleepiness, with effort to keep awake) }\end{array}$ & Avoidable crash zone \\
\hline
\end{tabular}




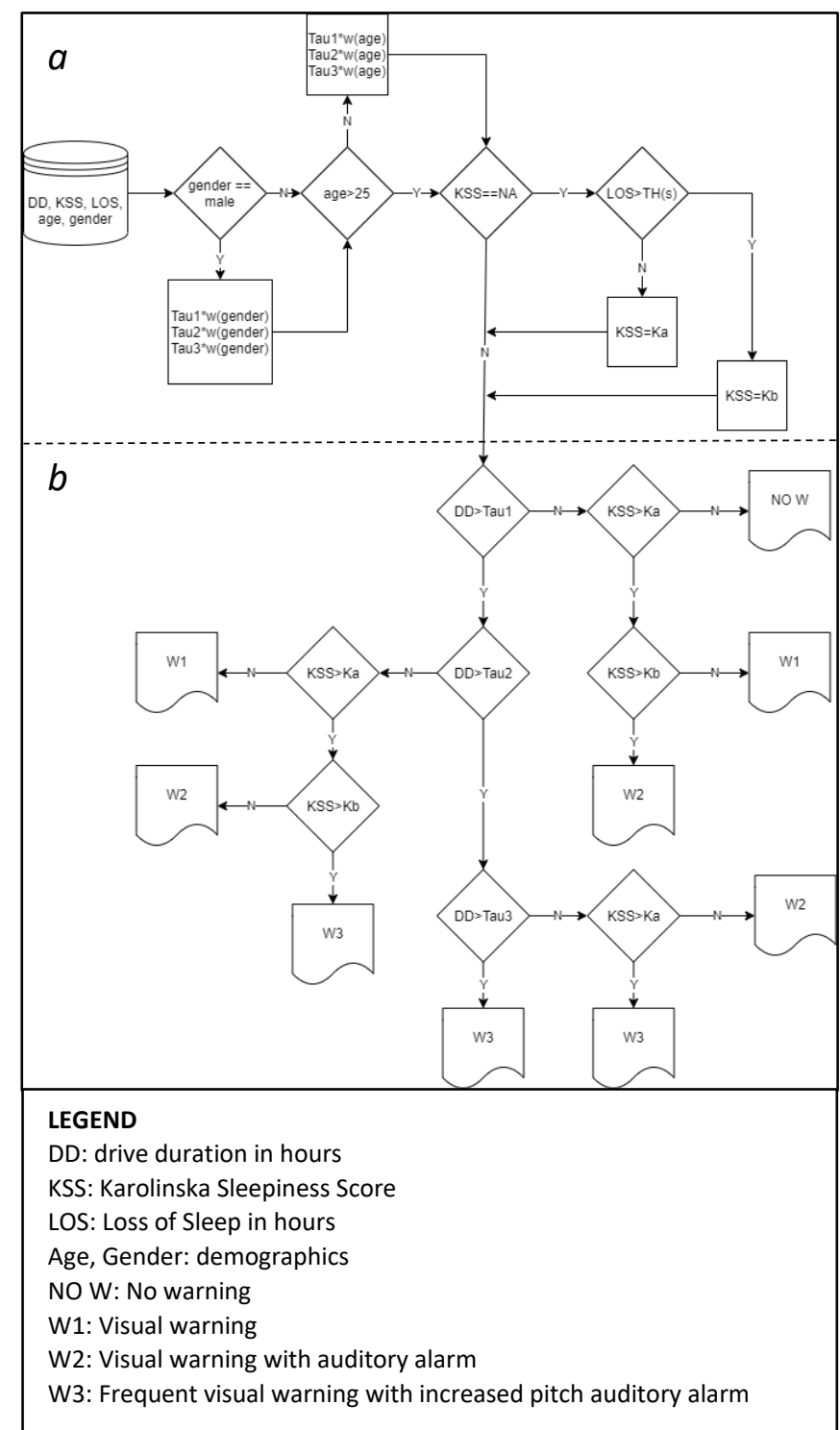

Fig. 2. The flowchart of fatigue warning strategies for passenger cars in iDREAMS

The visual warning is accompanied by an auditory alarm if the KSS score is more than $\mathrm{K}_{\mathrm{b}}-\mathrm{a}$ state that is assumed to be the "avoidable crash" phase of the STZ.

As the drive duration increases and falls between $h_{1}$ hours and $\mathrm{h}_{2}$ hours, a visual warning is given if the KSS score is less than $\mathrm{K}_{\mathrm{a}}$ (indicating the "danger" phase). This visual warning will be accompanied by an auditory alarm if the KSS score is between $\mathrm{K}_{\mathrm{a}}$ and $\mathrm{K}_{\mathrm{b}}$ (indicating the "avoidable crash" phase). The frequency of the visual warning and the pitch of the auditory alarm is increased if the KSS score of the drivers is more than $\mathrm{K}_{\mathrm{b}}$. If the drive duration is more than $\mathrm{h}_{2}$ hours but less than $h_{3}$ hours, a visual and auditory alarm is given to the drivers if the KSS score is less than $\mathrm{K}_{\mathrm{a}}$. The frequency of the visual warning and the pitch of the auditory alarm is increased if the KSS score is more than $\mathrm{K}_{\mathrm{a}}$. Finally, drive duration more than $\mathrm{h}_{3}$ hours may be associated with extreme high risk and so frequent visual warning with increased pitch auditory alarm is given to the drivers in these circumstances irrespective of the KSS score.

The above strategy clearly shows that the KSS score plays a critical role in providing warnings as part of the i-DREAMS fatigue intervention. However, as the KSS score is calculated based on the secondary measures of HRV obtained from sensors on the steering wheel, this may result in missing KSS scores if the secondary measures are not available (e.g., when driver's hands are not properly placed on the sensors). In such circumstances, the missing KSS score is replaced by its two thresholds depending on driver sleep duration and sleep loss, another indicator of sleepiness. Although the sleep loss is static i.e., does not change over the course of the drive, it still can be used as a proxy for sleepiness when the KSS score is missing. In such instances, if the sleep loss is less than a critical value, then the KSS score can be set to $K_{a}$ and otherwise it can be set to $\mathrm{K}_{\mathrm{b}}$. It is, however, challenging to obtain a reliable estimate of the critical value for sleep loss as this variable is relative to the usual sleeping hours of each individual. As a result, there should be baseline data collected to estimate a driver's typical sleep habits and average sleep duration.

\section{1) Varying thresholds for fatigue intervention}

The above-mentioned real-time i-DREAMS fatigue intervention strategy assumes that the STZ thresholds for each risk factor are fixed across all drivers and all driving conditions. This assumption, however, may not be entirely accurate. Due to the body's natural circadian rhythms causing increased sleepiness during the night, drive duration thresholds should be adjusted based on the time of the day (e.g., for daytime or night-time driving). In addition, younger and older drivers do not have the same driving ability (fitness) and so defining the same thresholds for these two age cohorts may not be entirely correct [5]. Distraction and bad weather conditions are among contextual factors that can increase mental workload and deteriorate fatigue in long periods of drive durations [18]. As such, it is necessary to adjust the thresholds based on demographics and driving conditions. It is, however, important to note that the KSS score is a selfreported measure of sleepiness and has a defined meaning. As a result, the adjustments are only applicable to drive duration as the measure of fatigue.

Informed by the international risk assessment program's (iRAP) star rating scores [19], a multiplicative approach is adopted for adjusting the thresholds in this study where the adjusted threshold ( $\tau_{\text {adjusted }}$ ) can be calculated as a product of an Adjustment Index (AdI ) and the original threshold $(\tau)$ :

$$
\tau_{\text {adjusted }}=A d I \times \tau
$$

where $A d I=\prod w_{i}$ and $w_{i}$ is the adjustment factor for variable $i$. These adjustment factors are more than 1 if the variables increase fatigue and are less than 1 if the variables reduce fatigue. These adjustment factors may be obtained or approximated from previous studies using their impact on fatigue. For example, assuming previous studies in driving fatigue have suggested that drivers younger than 25 years old are more likely to become fatigued by around $\beta \%$ and male drivers, on average, are associated with more fatigue by around $\alpha \%$, then the adjustment factors for age and gender would be $w_{\text {age }}=1-\beta \%$ and $w_{\text {gender }}=1-\alpha \%$, respectively. As such, the thresholds of drive duration should be adjusted with respect to these two adjustment factors (section a in Fig. 2).

As mentioned previously, another adjustment that needs to be considered is regarding the time of the day. This adjustment is essentially based on the KSS score or 
progression across each KSS score. As such, it is assumed that correlating drive duration with KSS score (thoroughly explained earlier) automatically captures the effects of driving at different times of the day.

\section{2) Consideration for other transport modes}

The above thresholds are all for passenger cars. The thresholds for the other transport modes should be adjusted with respect to the regulations for professional drivers. For example, according to the European Union [20], professional truck drivers have to take an uninterrupted break of at least 45 minutes after a driving period of 4.5 hours. As such, the last drive duration threshold (Tau3 in Fig.2) should be adjusted for professional drivers, accordingly.

\section{3) Implementation and technical issues}

The duration of the drive and the KSS score are the key parameters that are needed for implementing fatigue interventions. The duration of the drive can be easily obtained in real-time using vehicle ignition and Global Positioning System (GPS) devices that are already installed in the vehicle. Obtaining an estimate of KSS score in real-time, on the other hand, may be challenging because the KSS score is a selfreported measure. As such, it may not be practical to ask the drivers about their KSS score in real-time while driving. Within the i-DREAMS fatigue intervention strategy, the KSS score is calculated based on HRV, using sensors installed on the steering wheel, referred to as CardioWheel [21], or a wearable device. The acquisition will be based on the electrocardiogram (ECG) when CardioWheel is applicable, and on photoplethysmography (PPG) when the wearable is used. With both scenarios the time series of the RR peaks is calculated and HRV features extracted. Unlike most real-time feedback devices, these sensors focus their real-time monitoring algorithms on the driver state using non-intrusive techniques and infer driver's sleepiness. The KSS score may then be approximated in specified time intervals (e.g., every 2 minutes within a 5 minute window) [22].

\section{B. Post-trip fatigue intervention}

Whereas in-vehicle fatigue warnings aim to influence operator's immediate decision making while driving, e.g., by nudging the driver to take a break or stop driving, post-trip interventions aim at creating a sustainable behavioural change over time. Hence, post-trip interventions (i.e. coaching) are operational prior to or after a trip and are primarily meant to empower the driver to make appropriate decisions while driving. Within i-DREAMS, this driver coaching is organized by means of a smartphone app running on the driver's own mobile device with the aim to:

- increase the driver's knowledge and understanding of the risks of driving while fatigued or sleepy through the provision of easy-to-understand risk figures obtained from crash statistics and scientific research;

- provide insight into the driver's own driving behaviour under circumstances of fatigue or sleepiness by visualizing on a trip-map, episodes of driving while sleepy or fatigued and translating these results into meaningful sleepiness or fatigue scores;

- provide coping tips on how the driver can avoid or react to events of sleepiness or fatigue in advance of, or during, a trip (and hereby improve on their scores);
- challenge the driver to take up a personalized goal to improve their sleepiness or fatigue score in future trips and reward the driver for good performance by means of a system of gamification (e.g., badges, levels, leader board etc.).

In order to feed this system of driver coaching with personalized data, the estimated KSS score (based on heart rate data for sleepiness while driving) and trip-related characteristics, (including time of day of the trip and trip duration), as well as data of sleep duration obtained from a wearable, are collected and processed in a cloud back-end system to calculate aggregate scores for sleep deprivation and fatigue. These scores constitute the basis to evaluate, in an automatic way by the i-DREAMS post-trip processing and gamification framework, whether the driver needs online coaching (by means of the smartphone app) on the behavioural aspect of fatigue and sleepiness. For professional drivers, virtual coaching can be supplemented by offline coaching by the employer (e.g. driver coach), for example in the context of continuous driver education, or as a part of mandatory periodic training organized to obtain or keep the certificate of professional competence (CPC).

\section{CONCLUSIONS AND FUTURE WORK}

It is important that fatigue and sleepiness can be detected in safety critical situations, such as driving. The current paper focuses on the sleepiness and fatigue aspects of the iDREAMS project. Using the i-DREAMS platform to monitor sleepiness and fatigue levels and to alert drivers through interventions (both real-time and post-trip) that their driving performance will be impaired, has potential to improve safety. However, the issue is at what point does the intervention occur and what does the intervention look like.

By incorporating trip duration and driver sleepiness information, the i-DREAMS system aims to provide realtime interventions for drivers to prevent sleepy or fatigued driving. This is then enhanced through personalised post-trip feedback related to their fatigue and sleepiness levels, aiming to provide coping tips and challenges to help improve driving behaviour. Both the real-time and post-trip interventions can be adapted to suit different transport modes. The next stage of development will be to test the strategies in a series of simulator ( $n=30$ per mode) and field trials: car $(n=235)$, bus $(n=75)$, truck $(n=125)$ and rail $(n=70)$. An important future consideration may be the acceptance of warning alerts and compliance with interventions. Future research should also explore alertness warning frequency, for example, the longer a driver is in the upper range of the KSS bands (increased sleepiness), the more frequent/urgent the alerts should be. It is, however, important to note that the third type of fatigue warning (W3 in the flowcharts) already includes increased frequency and pitch which is believed to capture this consideration, at least to some extent.

\section{ACKNOWLEDGMENT}

This research was funded by the EU H2020 i-DREAMS project (Project Number: 814761) funded by European Commission under the MG-2-1-2018 Research and Innovation Action (RIA). www.idreamsproject.eu.

\section{REFERENCES}


[1] S. Bioulac et al., "Risk of motor vehicle accidents related to sleepiness at the wheel: a systematic review and meta-analysis," Sleep, vol. 40, no. 10, 2017.

[2] S. H. Bartley, and E. Chute, Fatigue and impairment in man, McGraw Hill, New York, 1947.

[3] W. C. Dement, and M. A. Carskadon, "Current perspectives on daytime sleepiness: the issues," Sleep: J Sleep Res Sleep Med, vol. 5, no. 2, p. 56-66, 1982.

[4] A. Williamson, D. A. Lombardi, S. Folkard, J. Stutts, T. K. Courtney, and J. L. Connor, "The link between fatigue and safety," Accid Anal Prev, vol. 43, no. 2, p. 498-515, 2011.

[5] A. J. Filtness, L. A. Reyner, and J. A. Horne, "Driver sleepinessComparisons between young and older men during a monotonous afternoon simulated drive," Biol. Psychol, vol. 89 , no. 3, p. 580-583, 2012.

[6] S. S. Smith, M. S. Horswill, B. Chambers, and M. Wetton, "Hazard perception in novice and experienced drivers: The effects of sleepiness," Accid Anal Prev, vol. 41, no. 4, p. 729-733, 2009.

[7] C. Anderson, and J. A. Horne, "Driving drowsy also worsens driver distraction," Sleep Med, vol. 14, no. 5, p. 466-468, 2013.

[8] S. Otmani, T. Pebayle, J. Roge, and A. Muzet, "Effect of driving duration and partial sleep deprivation on subsequent alertness and performance of car drivers", Physiol Behav, vol. 84, no. 5, p. 715-724, 2005.

[9] J. M. Cori, C. Anderson, S. S. Soleimanloo, M. L. Jackson, and M. E. Howard, "Narrative review: Do spontaneous eye blink parameters provide a useful assessment of state drowsiness?," Sleep Med Rev, vol. 45, p. 95-104, 2019.

[10] A. Persson, H. Jonasson, I. Fredriksson, U. Wiklund and C. Ahlström, "Heart Rate Variability for Classification of Alert Versus Sleep Deprived Drivers in Real Road Driving Conditions," IEEE trans Intell Transp Syst, 2020.

[11] J. Vicente, P. Laguna, A. Bartra, and R. Bailón, "Drowsiness detection using heart rate variability," Med Biol Eng Comput, vol. 54, p. 927 937,2016
[12] H. Lee, J. Lee, and M. Shin, "Using wearable ECG/PPG sensors for driver drowsiness detection based on distinguishable pattern of recurrence plots," Electronics, vol. 8, no. 2, p. 192, 2019.

[13] J. R. Pinto, J. S. Cardoso, A. Lourenço, and C. Carreiras, "Towards a continuous biometric system based on ECG signals acquired on the steering wheel," Sensors, vol. 17, no. 10, p. 2228, 2017.

[14] T. Åkerstedt, and M. Gillberg, "Subjective and objective sleepiness in the active individual," Int J Neurosci, vol. 52, no. 1-2, p. 29-37, 1990.

[15] C. Katrakazas et al., "Technologies for safety interventionsand assessment of their effectiveness", Deliverable 2.2 of the EC H2020 projecti-DREAMS, 2020.

[16] C. Ho, and C. Spence, "Assessing the effectiveness of various auditory cues in capturing a driver's visual attention,” J Exp Psychol Appl, vol. 11, no. 3, p. 157-174, 2005.

[17] D. R. Large et al., "Investigating the effect of urgency and modality of pedestrian alert warnings on driver acceptance and performance," Transp Res Part F Traffic Psychol Behav vol. 60, p. 11-24, 2019.

[18] L. C. Barr, C. D. Yang, R. J. Hanowski, and R. Olson, "Assessment of driver fatigue, distraction, and performance in a naturalistic setting," Transp Res Rec 1937, vol. 1, p. 51-60, 2005.

[19] IRAP, “iRAP Methodology Fact Sheet 6: Star Rating Score Equations," International Road Assessment Programme, 2014.

[20] A. Jensen, and S. Dahl, "Truck drivers hours-of-service regulations and occupational health," Work, vol. 33, no. 3, p. 363-368, 2009.

[21] A. Lourenço , A. P. Alves , C. Carreiras , R. Duarte , A. L. N. Fred, "CardioWheel: ECG biometrics on the steering wheel", European Conf. on Machine Learning and Principles and Practice of Knowledge Discovery in Databases - ECML/PKDD, Porto, Portugal, September, 2015.

[22] C. Pinto, J. Cardoso, A. Lourenço, C. Ahlstrom, "The importance of subject-dependent classification and imbalanced distributions in driver sleepiness detection in realistic conditions", IET Intelligent Transport Systems, Oct 2018 